\title{
P-0873 - Trend of unhealthy lifestyle behaviors and glycemia control among elderly with T2DM in rural China from 2014 to 2018
}

\author{
Shuling Liu' ${ }^{1,2}$, Xue Cai ${ }^{1}$, Hua Jiang ${ }^{1}$, Zhiying Ma ${ }^{1}$, Jun Li ${ }^{1}$, Mingzi Li ${ }^{1^{*}}$ \\ 1.School of Nursing, Peking University, Beijing, China; \\ 2.Department of Neurology, Tianjin Huanhu Hospital, Tianjin, China
}

\section{Background}

In China, most of DM patients are followed-up at diabetic clinics that are run by general practitioners and/or internist at primary health care centers that are spread across the country. However, there is limited data about unhealthy lifestyle behaviors and glycaemic control at primary health care centers in China.

\section{Aims}

To analyze the changes in of unhealthy lifestyle behaviors, glycemic control and pharmacotherapy of type 2 diabetes mellitus (T2DM) in Chinese rural primary health care center 2014-2018.

\section{Method}

This was a cross-sectional study carried out in rural primary health care center in northern China from 2014-2018. Type 2 diabetic outpatients were included. Data on general characteristics, unhealthy lifestyle behaviors(smoking, drinking, and $\mathrm{No} /$ Less Exercise), treatment regimen, complications and blood glucose levels were collected using a questionnaire interview. A glycated HbA1c of less than $7.0 \%$ was set as treatment target. Data were analyzed using descriptive statistics.

\section{Results}

From 2014 to 2018, 2356, 2551, 2400, 2610, 2719 elderly people were enrolled, including 251(10.65\%), 312 (12.23\%), $386(16.08 \%), 462(17.70 \%), 520(19.12 \%)$ patients with T2DM respectively. The rate with at least one concomitant disease in each year were $59.8 \%, 66.7 \%, 66.3 \%, 65.4 \%$, and $68.8 \%$, respectively. In 2018, the average HbA1c level was $7.82 \%$, in addition, the patients reaching the target of HbAlc $(<7 \%)$ was $29.2 \%$. The most common concomitant disease were hypertension. The unhealthy lifestyle behaviors accounted for $73.7 \%, 59.9 \%, 49.2 \%, 60.6 \%$, and $75.4 \%$, respectively.

\section{Discussion}

The overall rate of achieving HbA1c control target remained low among Chinese T2DM patients in rural areas. About 50\% of T2DM patients had at least one concomitant disease and unhealthy lifestyle behaviors. The study call for an early, comprehensive intervention strategies of diabetes in primary health care settings in rural China.

Keywords Diabetes mellitus, type 2; HbA1c; Lifestyle behaviors ; Glycemic control; Primary Health Care; Elderly

Table 1 Unhealthy behavior and glycemic control goals of T2DM patients conveyed from 2014 to 2018

\begin{tabular}{|c|c|c|c|c|c|}
\hline \multirow{2}{*}{ Characteristics } & \multicolumn{5}{|c|}{ Year } \\
\hline & 2014 & 2015 & 2016 & 2017 & 2018 \\
\hline DM patients & 251 & 312 & 386 & 462 & 520 \\
\hline Smoking (n [\%]) & $35(13.9)$ & $50(16.0)$ & $57(14.8)$ & $78(16.9)$ & $80(15.4)$ \\
\hline Drinking (n [\%]) & $14(5.6)$ & $26(8.3)$ & $35(9.1)$ & $55(11.9)$ & $64(12.3)$ \\
\hline No/Less Exercise & $169(67.3)$ & $152(48.7)$ & $146(37.8)$ & $233(50.4)$ & $358(68.8)$ \\
\hline $\begin{array}{l}\text { Unhealthy behavior } \\
\text { (any of three unhealthy } \\
\text { behavior) }\end{array}$ & $185(73.7)$ & $187(59.9)$ & $190(49.2)$ & $280(60.6)$ & $329(75.4)$ \\
\hline Totally unhealthy & $6(2.4)$ & $10(3.2)$ & $7(1.8)$ & $18(3.9)$ & $24(4.6)$ \\
\hline Hypertension, n (\%) & $150(59.8)$ & $208(66.7)$ & $256(66.3)$ & $302(65.4)$ & $358(68.8)$ \\
\hline Dyslipidemia, n (\%) & $97(38.7)$ & $117(37.4)$ & $150(38.9)$ & 181(39.3) & $196(37.8)$ \\
\hline HbA1c $($ Mean \pm SD, \%) & $7.76 \pm 2.46$ & $8.12 \pm 2.95$ & $7.69 \pm 1.85$ & $7.98 \pm 1.74$ & $7.82 \pm 2.45$ \\
\hline HbA1c $<7 \%$ & $62(24.7)$ & $84(26.8)$ & $97(25.1)$ & $139(30.0)$ & $152(29.2)$ \\
\hline$\geq 7 \%$ & $189(75.3)$ & $228(73.2)$ & $289(74.9)$ & $323(70.0)$ & $368(70.8)$ \\
\hline
\end{tabular}

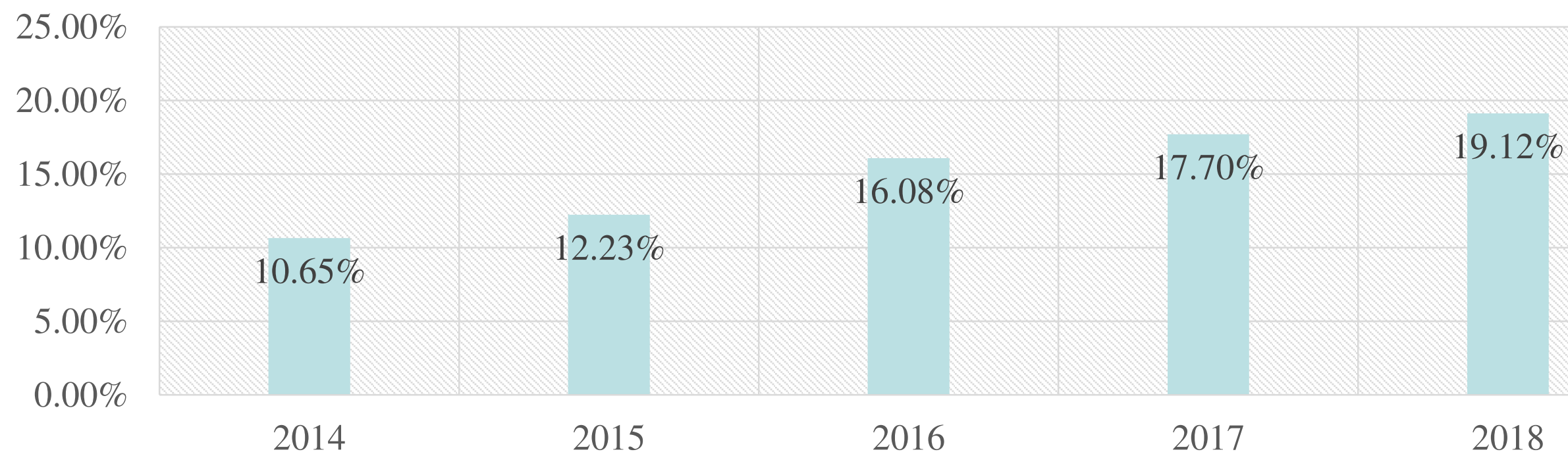

